\title{
Development and implementation of some BEM variants-a critical review
}

\begin{abstract}
Due to rapid development of boundary element method (BEM), this article explores the evolution of BEM over the past half century. We here summarize the overall development and implementation of several well-known BEM variants that includes collocation BEM, galerkin BEM, dual reciprocity BEM, complex variable BEM and analog equation method. Their theoretical and mathematical backgrounds are carefully described and a generalized

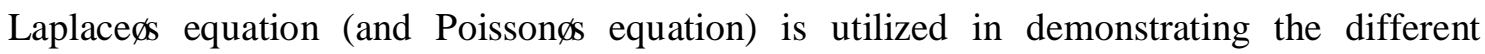
approaches involved. An up-to-date review on characteristics and implementation for each of the five variants is presented and also highlighted their significant contributions in boundary element research. In addition, this article tries to cover whole aspect of interests including efficiency, applicability and accuracy in order to give better understanding of BEM evolution. Comparisons and techniques of improvement for these variants are also discussed.
\end{abstract}

Keyword: Boundary element method (BEM); Collocation BEM; Galerkin BEM; Dual reciprocity BEM; Complex variable BEM; Analog equation method; BEM development; Review 